Original Paper http://ajol.info/index.php/ijbcs http://indexmedicus.afro.who.int

\title{
Dynamique spatio-temporelle des savanes boisées de la forêt classée de Tiogo au Burkina Faso
}

\author{
Boalidioa TANKOANO ${ }^{1 *}$, Mipro HIEN ${ }^{1}, \mathrm{~N}^{\prime}$ Da Hyppolite DIBI $^{2}$, \\ Zézouma $\mathrm{SANON}^{1}$, Jérôme T. YAMEOGO ${ }^{1}$ et Irénée SOMDA $^{1}$

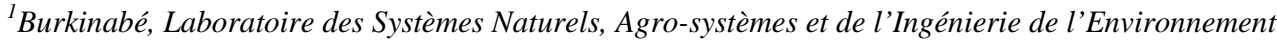 \\ (Sy.N.A.I.E), Institut du Développement Rural, Université Polytechnique de Bobo-Dioulasso, \\ 01 BP 1091 Bobo-Dioulasso 01 Burkina Faso. \\ ${ }^{2}$ Centre Universitaire de Recherche et d'Application en Télédétection, UFR Biosciences, Université Félix \\ Houphouët Boigny Abidjan-Cocody, 23 BP 4957 Abidjan 23, République de Côte d'Ivoire. \\ *Auteur correspondant, E-mail : btankus67@yahoo.fr, Tél: +22670570438/76558795
}

\section{RESUME}

Les aires protégées du Burkina Faso connaissent un phénomène d'anthropisation accrue. A cela s'ajoute l'effet des changements climatiques. Ainsi, ces facteurs ont des conséquences dont la baisse de la biodiversité. Cette étude a pour objectif principal d'analyser de la dynamique spatio-temporelle des savanes boisées. Elle a été conduite dans la forêt classée de Tiogo. Des images Landsat TM de 1986 et ETM de 2010 ont été utilisées. Deux cartes d'occupation du sol, respectivement de 1986 et de 2010 ont été réalisées avec trois classes thématiques. De ces deux cartes d'occupation du sol, deux cartes de répartition spatiale des savanes boisées de 1986 et 2010 ont été générées. Les résultats cartographiques révèlent une forte régression des savanes boisées passant de 35,17\% en 1986 à 18,04\% en 2010. Durant la même période, les zones d'anthropisation ont progressé d'environ 4,38\% par an. L'analyse de la dynamique spatiale a montré une fragmentation des savanes boisées entre 1986 et 2010. Cette fragmentation est principalement due à des activités anthropiques, notamment l'exploitation forestière et les défrichements agricoles. Ceci constitue donc une menace croissante pour la conservation de la biodiversité dans cette forêt classée si aucune mesure n'est prise pour inverser les tendances.

(c) 2015 International Formulae Group. All rights reserved.

Mots clés : Aires protégées, savanes, fragmentation, anthropisation, imagerie satellitaire, Afrique de l'Ouest.

\section{Spatio-temporal dynamics of woody savannahs in the Tiogo protected area of Burkina Faso}

\begin{abstract}
This study is a contribution to the analysis of the spatio-temporal dynamics of the savanna within protected areas. It was conducted in the classified forest of Tiogo in Central West of Burkina Faso. Images 1986 Landsat TM and ETM of 2010 have been used. Two land use maps of 1986 and 2010 respectively, were made with respect to three thematic classes defined. From these two land use maps, two maps of spatial
\end{abstract}


distribution of woody savanna of 1986 and 2010 were generated. The mapping results reveal a sharp decline of woody savanna, declining from $35.17 \%$ in 1986 to $18.04 \%$ in 2010 . During the same period, anthropization areas have increased of approximately $4.38 \%$ per year. Analysis of the spatial dynamics has shown fragmentation of savanna between 1986 and 2010. This fragmentation is due to anthropogenic activities, including logging and agricultural clearing. This is therefore a growing threat to the conservation of biodiversity in this classified forest if no action is taken to reverse the trends.

(C) 2015 International Formulae Group. All rights reserved.

Keywords: Protected areas, savannas, fragmentation, anthropization, satellite imagery, West Africa.

\section{INTRODUCTION}

La forêt reste un bien commun universel et un écosystème complexe (Kabulu et al., 2008). Pour la même source, la forêt contribue à l'équilibre de la nature et du climat. Elle sert d'habitat à un grand nombre d'espèces animales et végétales et remplit également de nombreuses autres fonctions comme le contrôle de l'érosion, le stockage de l'eau, la séquestration du carbone, l'interception et la redistribution des précipitations. Malheureusement, la plupart des paysages de par le monde sont modifiés par les activités humaines engagées pour répondre aux besoins socio-économiques des populations (Fahrig, 2003; Kabulu et al., 2008; Inoussa et al., 2011 ; Toko et al., 2012). Les aires protégées sont ainsi de plus en plus sous la pression humaine et subissent les péjorations climatiques. En effet, les aires protégées du Burkina Faso qui constituent les réservoirs de biodiversité n'échappent pas à ces phénomènes. Selon DIFOR (2007) le Ministère de l'environnement et du cadre de vie estime que $60 \%$ des aires du domaine classé sont occupées par des exploitations agricoles, des hameaux de cultures et mêmes des villages dotés d'infrastructures socioéconomiques. Les occupants de ces aires y pratiquent des feux de végétation, la coupe illégale du bois, le surpâturage et la carbonisation. A cela s'ajoutent les effets du changement global du climat (Inoussa et al., 2011). Pour certains auteurs, en Afrique de l'Ouest, les formes dominantes de perturbations de la structure et de la physionomie de la végétation au sein des aires protégées sont les feux de végétation; le pâturage, l'agriculture itinérante sur brulis et l'irrégularité de la pluviométrie (Hien et al., 2002; Grégroire et Simonetti, 2010). Ainsi, les effets combinés de ces deux facteurs (activités humaines et changement climatique) conduisent à la fragmentation d'écosystèmes forestiers et à la perte d'habitats de la faune et de la flore (Touré, 2010 ; Inoussa et al., 2011 ; Mama et al., 2013). Ces phénomènes sont une menace pour la gestion de la biodiversité et le fonctionnement des écosystèmes dans les aires protégées (Clerici et al., 2007).

Bien que la pression sur les ressources naturelles ne cesse de s'accentuer, des données précises sur la dynamique de la végétation et sur l'appauvrissement de la biodiversité de certaines formations forestières font encore défaut au Burkina Faso. Pourtant, ces informations sont d'une importance capitale pour une prise de conscience du phénomène et l'élaboration de plan d'aménagement forestier visant une exploitation rationnelle des ressources végétales. En plus, il est désormais question de mieux gérer le couvert végétal, dans l'optique de la séquestration de carbone (Mbow, 2009). C'est dans ce contexte que cette étude a été initiée. L'objectif général est d'analyser la dynamique spatio-temporelle des savanes boisées de la forêt classée de Tiogo par l'imagerie satellitaire. De façon spécifique, il s'agit de : cartographier la forêt classée de Tiogo en 1986 et en 2010 à partir des images Landsat; faire une répartition spatiale des îlots de savanes boisées et leur dynamique entre 1986 et 2010 et calculer les indices de structure spatiale des îlots de savanes boisées en 1986 et en 2010. 


\section{MATERIEL ET METHODES}

Description de la zone d'étude

Classée en 1940 par l'administration coloniale, la forêt classée de Tiogo couvre une superficie de 30669 ha. La zone d'étude est précisément localisée entre les longitudes $2^{\circ} 42^{\prime}$ et $2^{\circ} 52^{\prime}$ Ouest et les latitudes $12^{\circ} 13^{\prime}$ et $12^{\circ} 24^{\prime}$ Nord (Figure 1). Le relief est plat et monotone dans son ensemble, avec un certain nombre de buttes cuirassées (Sawadogo, 2009). L'altitude moyenne est de $300 \mathrm{~m}$. Au niveau pédologique, la Forêt Classée est caractérisée par des sols peu profonds, des sols hydromorphes et des sols à sesquioxyde de fer, avec des profondeurs variables (Nouvellet et Sawadogo, 1995). La zone d'étude est sous un climat de type soudanien, caractérisé par une saison sèche et une saison de pluies. La température moyenne annuelle est de $24{ }^{\circ} \mathrm{C}$, avec une amplitude thermique de $15^{\circ} \mathrm{C}$ (Savadogo, 2002).

Le potentiel hydrographique de la forêt classée repose essentiellement sur le fleuve Mouhoun auquel s'ajoutent des rivières et marigots temporaires. Les activités socioéconomiques sont l'agriculture extensive, l'élevage et l'exploitation forestière (Sawadogo, 2009).

\section{Images satellitaires et logiciels utilisés}

Les images satellitaires utilisées sont une scène Landsat 5 TM (Path 196, Row 51) du 08 octobre 1986 et une scène Landsat 7 ETM+ (Path 196, Row 51) du 27 novembre 2010. Elles ont été acquises à la même période de l'année afin de réduire les problèmes liés aux différences d'angles solaires, aux changements phénologiques de la végétation et à la différence d'humidité des sols. Toutes ces images sont issues de la base de données de l'United State Geological Survey (USGS). Les logiciels Envi 4.2 et ArcGis 9.3 ont été respectivement utilisés pour le traitement numérique des images et pour la cartographie.

\section{Traitement numérique et cartographie}

La première opération a consisté à extraire de l'image Landsat, une fenêtre d'étude centrée sur la forêt classée en utilisant le fichier «shapefile» du contour de la forêt classée. La deuxième opération a concerné l'amélioration de l'image en vue de faciliter la discrimination des types d'occupation du sol. Des compositions colorées 4-3-2 ont ensuite été réalisées sur les bandes brutes de Landsat5 TM et de Landsat7 ETM+. Ainsi, différents types d'occupation du sol comme les savanes boisées, les champs et jachères, les sols nus, les habitations et les autres types de formation végétale (savanes arbustives et arborées claires), ont été distinguées. Pour confirmer ces premiers résultats, quatre indices biophysiques ont été calculés. Il s'agit de l'indice de brillance des sols (Brightness Index), l'indice d'humidité (Wetness Index), l'indice de verdure (Greenness Index) et l'indice de végétation normalisé (Normalized Difference Vegetation Index). Ces indices ont permis de mettre en évidence :

- le niveau de recouvrement ou densité de la couverture végétale (BI) selon la formule : $\mathrm{BI}=0,3037 \mathrm{Band} 1+0,2793$ Band $2+$ 0,4743 Band $3+0,5585$ Band $4+0,5082$ Band 5 $+0,1863$ Band7

- le niveau d'humidité de la végétation ou du sol sous-jacent (WI) selon la formule :

$\mathrm{WI}=0,1509$ Band $1+0,1973$ Band $2+$ 0,3279 Band $3+0,3406$ Band $4-0,7112$ Band5 $-0,4572$ Band 7

- le niveau de stress hydrique de la végétation (GI) selon la formule :

$\mathrm{GI}=-0,2848 \mathrm{Band} 1-0,2435 \mathrm{Band} 2-$ 0,5436 Band $3+0,7243$ Band $4+0,0840$ Band 5 $-0,1800$ Band 7

- et l'intensité de l'activité photosynthétique (NDVI) selon la formule : NDVI $=($ Ban4 - Band 3$) /($ Band $4+$ Band 3$)$. Sur la base des compositions colorées, des indices biophysiques et des documents cartographiques, 100 sites de « vérité terrain » ont été choisis dont 30 pour les savanes boisées, 46 pour les autres types de formations végétales et 24 pour les zones anthropisées (champs, sols nus et habitations). 
Cette phase de «vérité terrain» a été effectuée lors d'une mission courant octobrenovembre 2013 dans la forêt et sa périphérie. L'objectif de cette mission a été de faire une description des différents types de végétation et de noter le niveau de dégradation de la forêt. Les parcelles de «vérité terrain» sont caractérisées par leur homogénéité et leur taille supérieure ou égale à $900 \mathrm{~m}^{2}$.

En se basant sur les informations collectées sur le terrain, la classification dirigée par l'algorithme Maximum de vraisemblance a été appliquée. Et, elle a permis de produire les deux cartes d'occupation du sol de la forêt classée de Tiogo. Au total, 50 parcelles d'entraînement ont servi de base à cette classification dirigée et les 50 autres parcelles ont servi de contrôle.

La matrice de confusion de cette classification a été faite sur la base des parcelles de contrôle issues des parcelles de «vérité terrain», autres que celles ayant servi pour l'entrainement. Celle-ci a permis de mettre en évidence la performance du traitement et la fiabilité des résultats obtenus. Cette même technique a été reprise pour l'évaluation de la classification dirigée de 1986. Mais pour cette dernière, la carte d'occupation du sol de 2010, celle de la Base de Données sur l'Occupation des Terres (BDOT) de 2002 et les données de terrain de 2013 ont servi de vérité-terrain. Les parcelles d'entraînement et de contrôle sélectionnées sur les différentes compositions colorées de 1986 ont été faites dans les zones n'ayant pas subi de changements. Finalement, un filtre médian a été appliqué à toutes les images classifiées en vue de les rendre plus nettes par élimination des pixels isolés. Ensuite, leur vectorisation a été réalisée. Après cette étape, le fichier a été exporté en format «shapefile» pour être utilisé dans le logiciel ArcGis 9.3. Ce fichier, une fois édité, a permis de générer les cartes d'occupation du sol de la forêt classée de Tiogo en 1986 et en 2010 ainsi que les cartes de répartition spatiale des savanes boisées.
Dynamique des unités d'occupation du sol

Cette évaluation a permis d'analyser l'évolution des différents éléments de l'occupation du sol dans la forêt classée de Tiogo entre 1986 et 2010. Le taux moyen annuel d'évolution de chaque élément de l'occupation du sol a été calculé suivant la formule :

$$
T_{x}=\left[\left(\left(s_{2010}-s_{1986}\right) / s_{1986}\right) \times 100\right] / 24
$$

$\mathrm{T}_{\mathrm{x}}$ : Taux d'évolution moyen annuel; $\mathrm{S}_{1986}$ : superficie de la classe $\mathrm{i}$ en $1986 ; \mathrm{S}_{2010}$ : superficie de la classe i en $2010 ; 24$ : nombre d'années entre 1986 et 2010.

\section{Calcul des indices de structure spatiale des taches de savanes boisées}

Le calcul de ces indices de paysage a permis d'analyser la structure spatiale des savanes boisées entre 1986 et 2010. Ces indices ainsi calculés concernent :

\section{$\checkmark \quad$ Nombre de taches $\left(\mathrm{n}_{\mathrm{j}}\right)$}

Le nombre de taches $n_{j}$ de chaque classe $j$ a été déterminé. Cet indice nous renseigne sur la fragmentation d'une classe entre deux périodes. En effet, l'augmentation du nombre de taches d'une classe peut être due à la fragmentation de cette classe (Davidson, 1998).

\section{$\checkmark \quad$ Aire totale $\left(\mathrm{a}_{\mathrm{tj}}\right)$}

L'aire totale $\left(a_{t j}\right)$ occupée par la classe $j$ (ha) a été calculée suivant l'équation cidessous où $a_{i j}$ est l'aire de la $i$-ème tache de la classe $j$ :

$a_{i j}=\sum_{i=1}^{n_{j}} a_{i j}$

Si l'aire d'une classe est grande, sa fragmentation est censée être faible, et si elle est petite sa fragmentation est importante (Kabulu et al., 2008).

\section{$\checkmark \quad$ Dominance (Dj)}

La dominance $\operatorname{Dj}(\mathrm{a})$ indiquant la proportion d'aires occupées par la tache dominante dans la classe $\mathrm{j}$ a été aussi calculée suivant la formule ci-dessous : 


$$
D_{j}=\frac{a_{m a x_{j}}}{a_{t j}} \times 100
$$

Elle varie de $0 \leq D_{j}(a) \leq 100$. Plus la valeur de dominance est élevée, moins la classe sera fragmentée.

$\checkmark \quad$ Aire moyenne $\left(\bar{\alpha}_{j}\right)$

L'aire moyenne $a_{j}$ (la valeur moyenne de l'aire des taches de la classe $j$ ) a été calculée selon la formule suivante :

$\bar{a}_{j}=\frac{a_{t j}}{n_{j}}$

Cet indice sera d'autant plus grand que la classe présente en moyenne des blocs forestiers importants (Kabulu et al., 2008). On pourra donc espérer que le paysage sera d'autant plus intact que l'indice est plus élevé.

$\checkmark \quad$ Indice de forme moyenne $\left(\overline{I F}_{j}\right)$

La forme moyenne $\left(\overline{I F}_{j}\right)$ de la classe $j$

a été calculée par la formule :

$\overline{I F}_{j}=\sum_{i=1}^{n_{j}} \frac{I F_{i j}}{n_{j}}$

Où l'indice de forme $I F_{i j}$ de la i-ème

tache de la classe $j$ est obtenu par le rapport de l'aire $a_{i j}$ de la tache $i$ de la classe $j$ sur le

carré de son périmètre $p_{i j}$ (Patton, 1975)

$$
I F_{i j}=\frac{a_{i j}}{p_{i j}^{2}}
$$

\section{Taux de fragmentation}

Le taux de fragmentation de la classe $\mathrm{j}$ sera calculé par la formule :
$F_{j}=\frac{n_{j}-1}{m_{j}-1}$

Où $m_{j}$ est, généralement dans un fichier raster, le nombre de pixels dans la classe $j$. Il varie de $0 \leq F_{j} \leq 1$. La classe

est moins fragmentée si $F_{j}$ est proche de 0 et davantage fragmentée si $F_{j}$ est proche de 1 (Kabulu et al., 2008).

$\checkmark \quad$ Diversité des aires de taches

La diversité des aires des taches de la classe $j$, notée $H_{j}(a)$, a été calculée suivant la formule de l'indice de Shannon ci-dessous (Bogaert et Mahamane, 2005) où, ln représente le logarithme népérien.

$H_{j}(\alpha)=\sum_{i=1}^{n_{j}}-\left(\frac{a_{i j}}{a_{i j}} \ln \frac{a_{i j}}{a_{i j}}\right)$

La valeur de $H_{j}(a)$ varie de

$$
0 \leq H_{j}(a) \leq \ln \left(n_{j}\right)
$$

Cet indice mesure la

diversité relative des taches au niveau de la classe. Il est égal à 0 lorsque la classe n'est constituée que d'une seule tache et sa valeur va croître avec le nombre de taches et avec l'équitabilité entre les aires des taches de la classe (McGarigal et Marks, 1995).

L'équitabilité de Piélou des aires des taches de la classe $j \ldots$ ) se calcule par la formule :

$$
\begin{gathered}
E_{j}(a \\
E_{j}(a)=\frac{H_{j}(a)}{\ln \left(n_{j}\right)}
\end{gathered}
$$

La valeur de l'équitabilité de Piélou varie de $0 \leq E_{j}(a) \leq 1$. Elle est faible si la

classe comprend des taches dominantes et elle est élevée s'il y a des taches de taille similaire. 


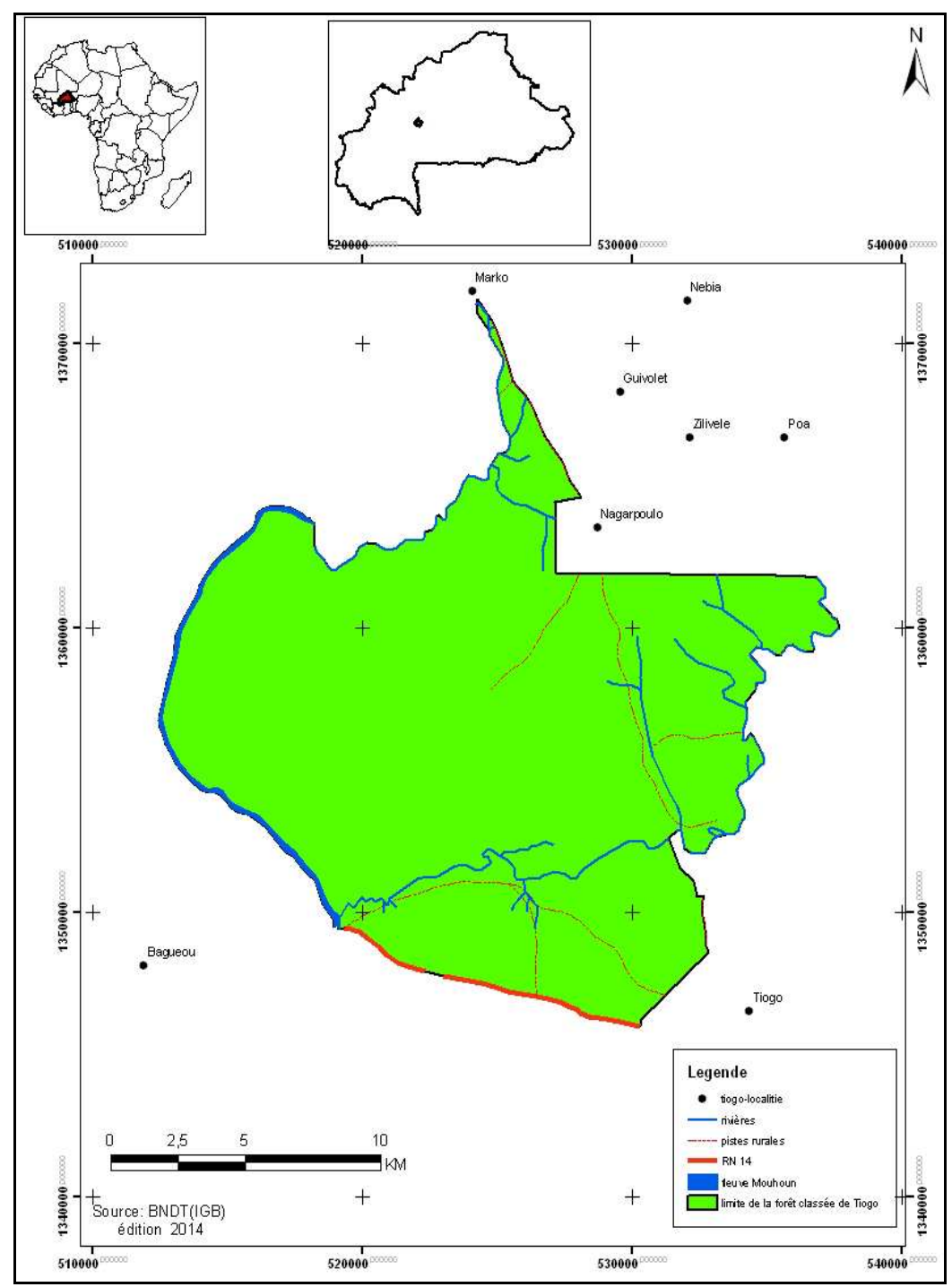

Figure 1 : Localisation de la Forêt Classée de Tiogo (Burkina Faso).

\section{RESULTATS}

\section{Vérification de la classification}

Les résultats de l'analyse de performance donnent pour chacune des images classifiées les précisions globales de $89,40 \%$ à $90,34 \%$ respectivement pour 1986 et 2010. Les matrices de confusion montrent globalement qu'il n'existe pas d'énormes confusions entre les classes tant lors de la classification de l'image de 1986 que celle de 2010. En plus, nous constatons que les savanes boisées (Photo 1) sont bien distinctes des autres classes d'occupation du sol. Pour les deux images, plus de $80 \%$ des pixels de la classe savanes boisées sont bien classés.

Etats d'occupation du sol en 1986 et en 2010

Les savanes boisées, les autres types de formation végétale et les zones 
anthropisées sont les trois classes d'occupation du sol qui ont finalement été retenues. Les cartes d'occupation du sol ont été générées avec une mise en relief des savanes boisées (Figures 2 et 3). Celles-ci illustrent spatialement et quantitativement les changements importants survenus dans cette forêt classée entre 1986 et 2010. La cartographie de l'occupation du sol en 1986 montre que les savanes boisées représentaient 10768 ha, soit $35,17 \%$ de la forêt classée. Ceci dénotait une présence remarquable de ladite classe dans cette aire protégée. Avec 3183 ha, les zones anthropisées représentaient 10,38\% de ladite forêt (Figure 2). Avec 5534 ha en 2010, les savanes boisées n'occupent plus que 18,04\% de la superficie de la Forêt Classée contre 35,17\% en 1986. Les zones d'anthropisation, avec une superficie de 6529 ha, occupent 21,29\% de l'ensemble de la Forêt Classée (Figure 3).

Dynamique des classes d'occupation du sol entre 1986 et 2010

Les résultats cartographiques et statistiques obtenus permettent de mettre en exergue la dynamique des classes d'occupation du sol entre 1986 et 2010 (Figure 4). Les zones anthropisées ont progressé de 3183 ha en 1986 à 6529 ha en 2010, soit une augmentation de 139,45 ha/an. Nous constatons que la classe «autres types de formations végétales » a également augmenté en superficie, passant ainsi de 16 699 ha en 1986 à 18605 ha en 2010. Contrairement aux deux autres classes d'occupation du sol; la classe des savanes boisées a reculé entre 1986 et 2010. Les savanes boisées ont perdu 5252 ha entre 1986 et 2010, soit un taux de régression annuel de $2,03 \%$. Nous avons aussi noté une exploitation fréquente du bois énergie (Photo 3) dans la forêt et d'autres activités anthropiques (Photos 4 et 5).
Répartition spatiale des taches de savanes boisées

Deux cartes de répartition spatiale des taches de savanes boisées (Figures 5 et 6 ) ont été générées pour1986 et pour 2010. Elles ont permis de mettre en exergue la distribution spatiale et temporelle des taches de savanes boisées.

\section{Indices de structure spatiale des savanes boisées}

Le Tableau 1 récapitule les indices de structure spatiale calculés pour la classe des savanes boisées en 1986 et en 2010. Ces indices ont permis de détecter les changements de la structure spatiale des îlots de savanes boisées de la forêt classée entre les deux dates. Le nombre de taches $\left(n_{j}\right)$ a augmenté entre 1986 et 2010 en passant de 559 à 1242. Ceci indique une fragmentation avec un morcellement des taches initiales. La tendance à la fragmentation est confirmée par la diminution de l'aire moyenne ( ${ }^{-}$aj) des taches de savanes boisées entre 1986 et 2010 . La valeur de l'aire moyenne de la classe a considérablement chuté, elle passe de 19,3 ha en 1986 à 4,46 ha en 2010. La dominance (Dj (a) a diminué de façon spectaculaire, passant de 45,57\% en 1986 à 4,46\% en 2010. Donc nous constatons qu'en 24 ans, les taches qui dominaient cette classe de savanes boisées ont été morcelées environ cinq (05) fois. Le taux de fragmentation $\left(F_{j}\right)$ des savanes boisées a connu une progression énorme entre les deux années. Le taux de fragmentation est passé de 0,005 en 1986 à 0,02 en 2010. Ce qui signifie que le taux de fragmentation a été multiplié par quatre (04) en 24 ans. L'anthropisation est également révélée par l'évolution de l'indice de forme $\left(\overline{\mathbf{I F}}_{\mathrm{j}}\right)$. La valeur de l'indice de forme

a augmenté et est passée de 0,005 en 1986 à 0,038 en 2010. Nous constatons que pour ces taches de savanes boisées, la valeur de 
l'indice de Shannon a augmenté entre 1986 et 2010. Elle passe de 2,89 en 1986 à 5,21 en 2010. Cela montre qu'en 1986, les aires des taches de savanes boisées étaient disproportionnelles. La forte valeur de l'indice de Shannon en 2010, traduit une augmentation du nombre de taches pour ladite classe. Quant à l'indice d'équitabilité de Piélou, il est passé de 0,46 en 1986 à 0,73 en 2010. Ceci montre qu'en 1986, il y avait un déséquilibre entre les aires des taches de la classe de savanes boisées. Par contre, la valeur de l'équitabilité de Piélou en 2010 met en relief un certain équilibre entre les aires des taches de la classe considérée.

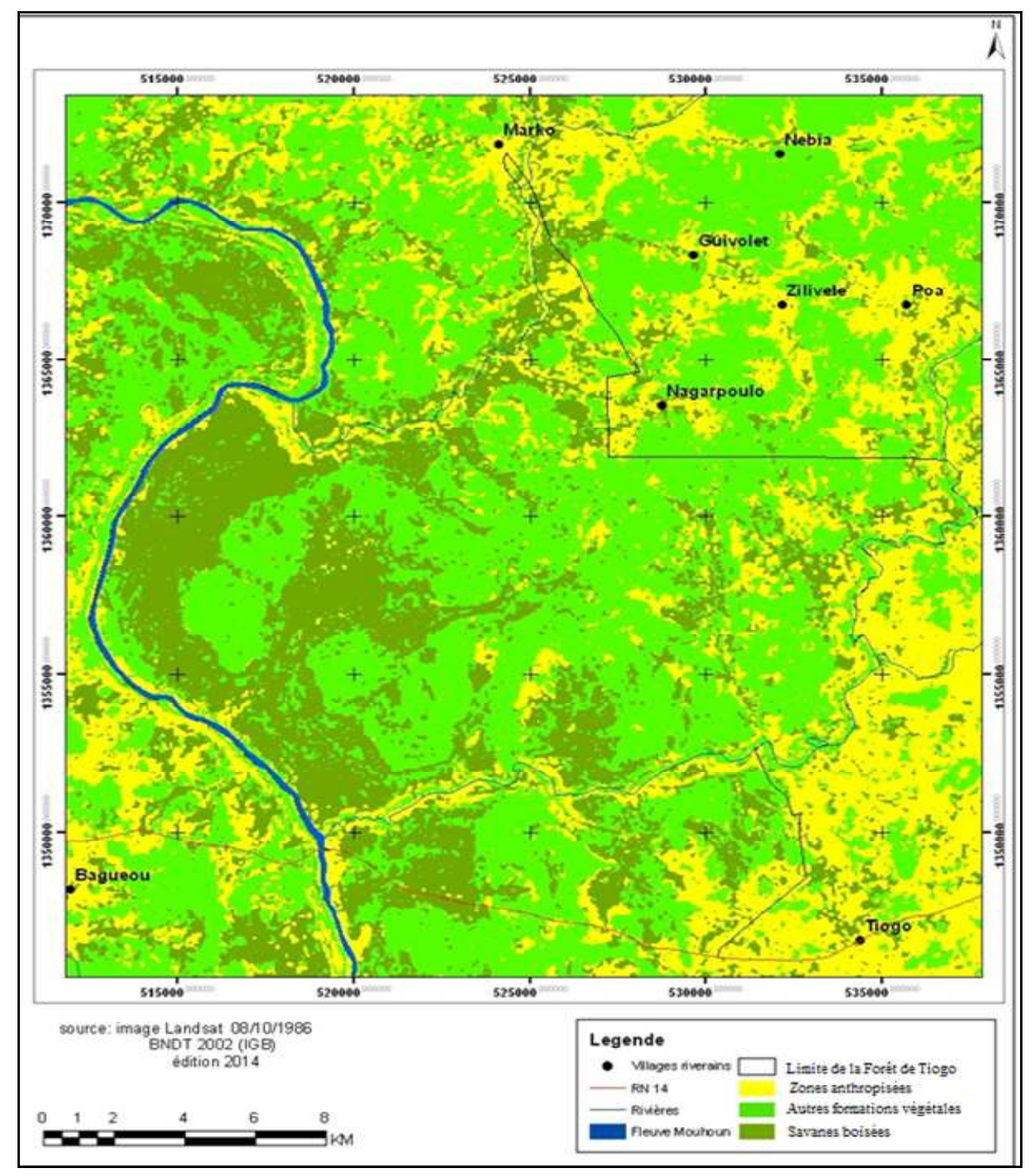

Figure 2 : Carte d'occupation du sol de la Forêt Classée de Tiogo et sa périphérie en 1986. 


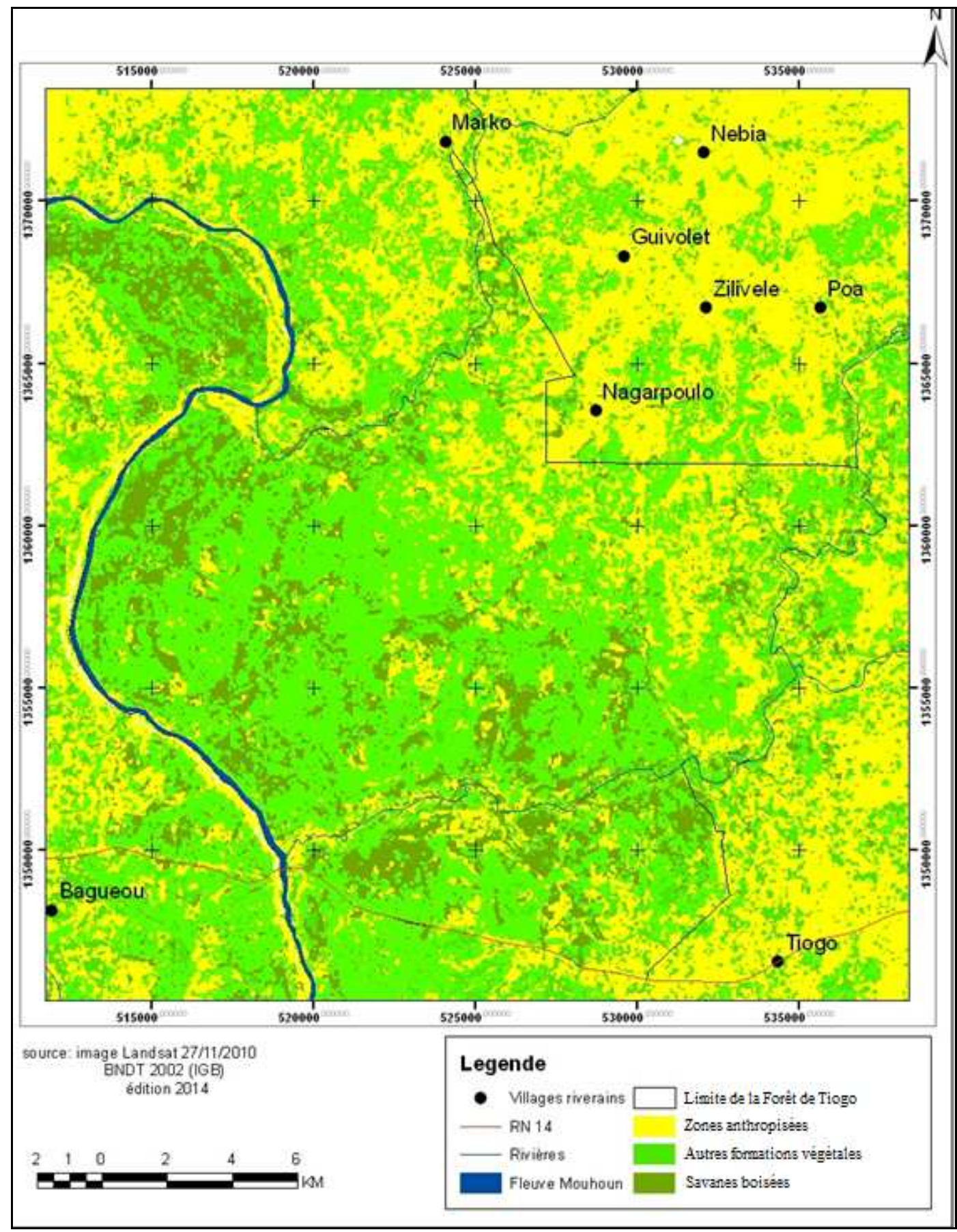

Figure 3 : Carte d'occupation du sol de la Forêt Classée de Tiogo et sa périphérie en 2010. 


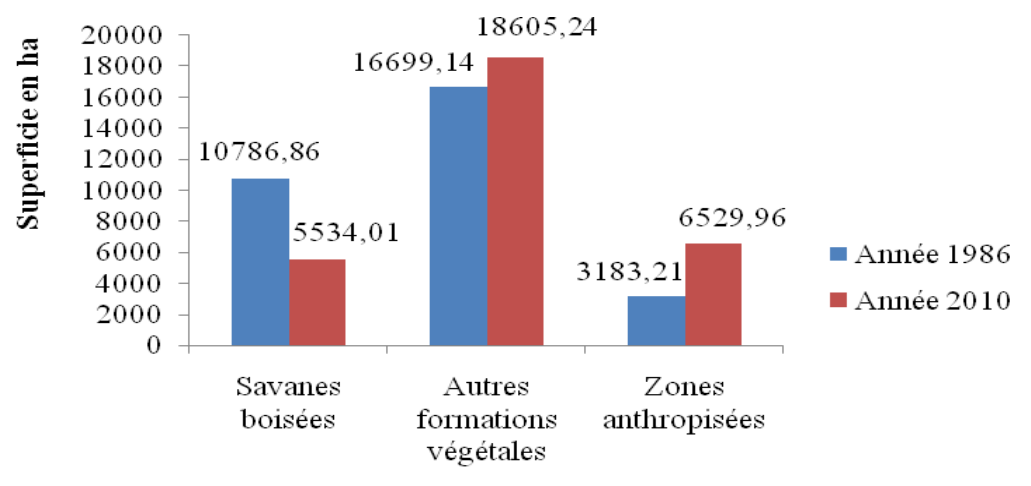

Classes d'occupation du sol

Figure 4 : Dynamique des classes d'occupation du sol de la Forêt Classée de Tiogo entre 1986 et 2010.

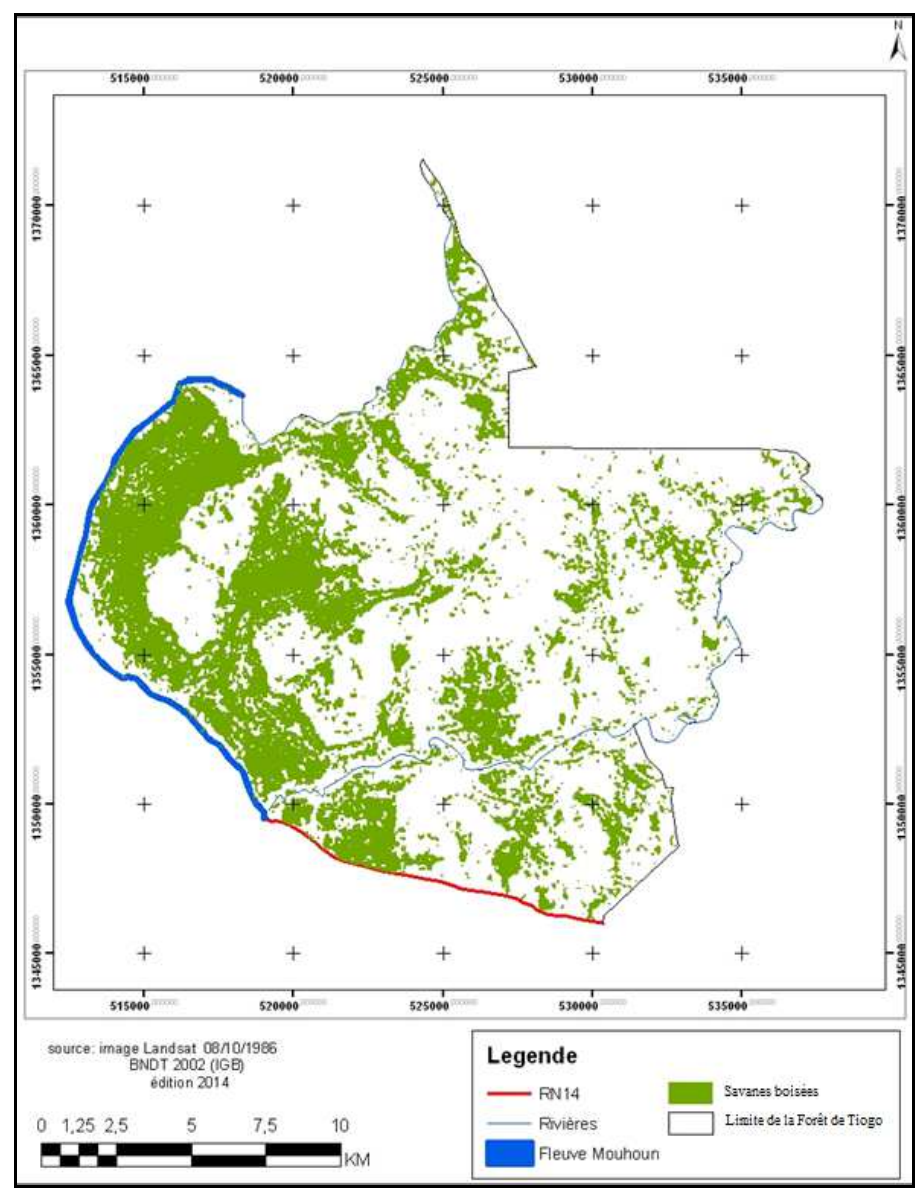

Figure 5 : Carte des taches de savanes boisées de la Forêt Classée de Tiogo en 1986. 


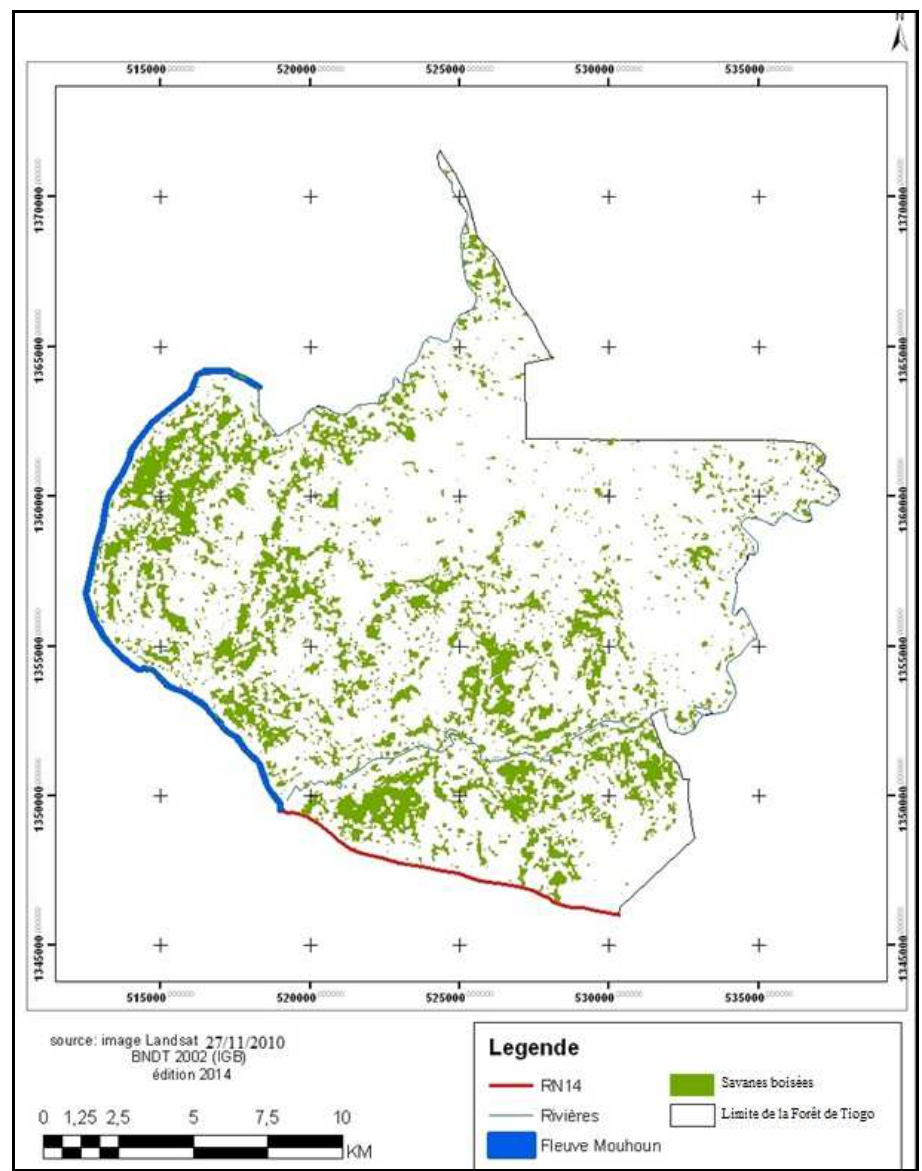

Figure 6 : Carte des taches de savanes boisées de la Forêt Classée de Tiogo en 2010.

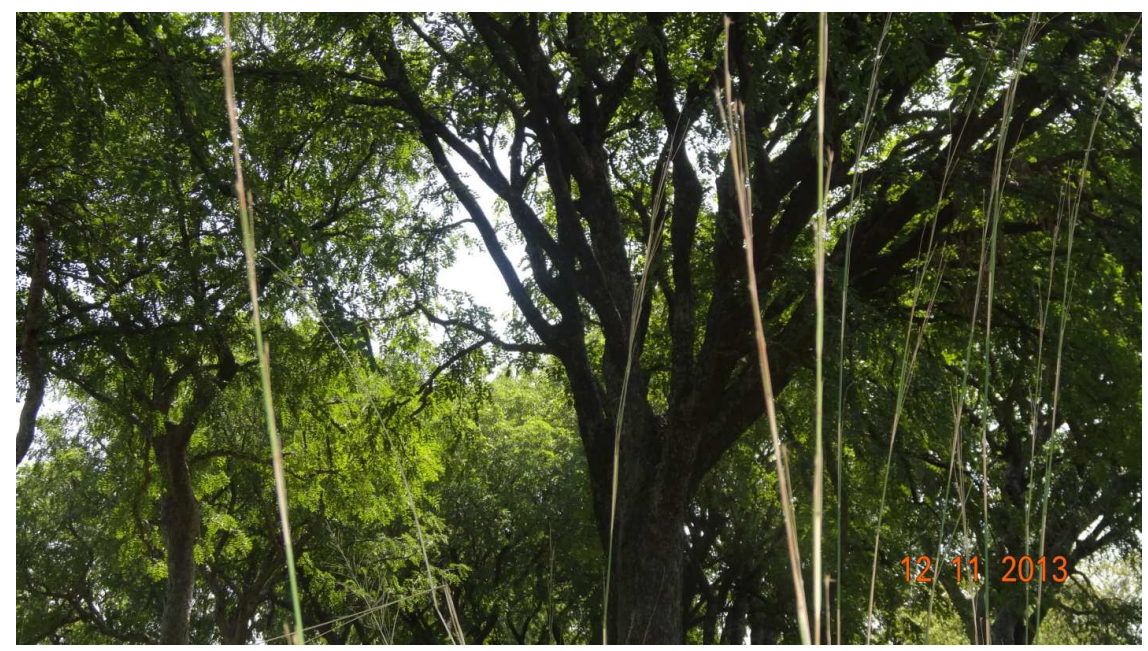

Photo 1 : Illustration des savanes boisées de la Forêt Classée de Tiogo (Tankoano, 2013). 1993 
P. BAZONGO et al. / Int. J. Biol. Chem. Sci. 9(4): 1983-2000, 2015

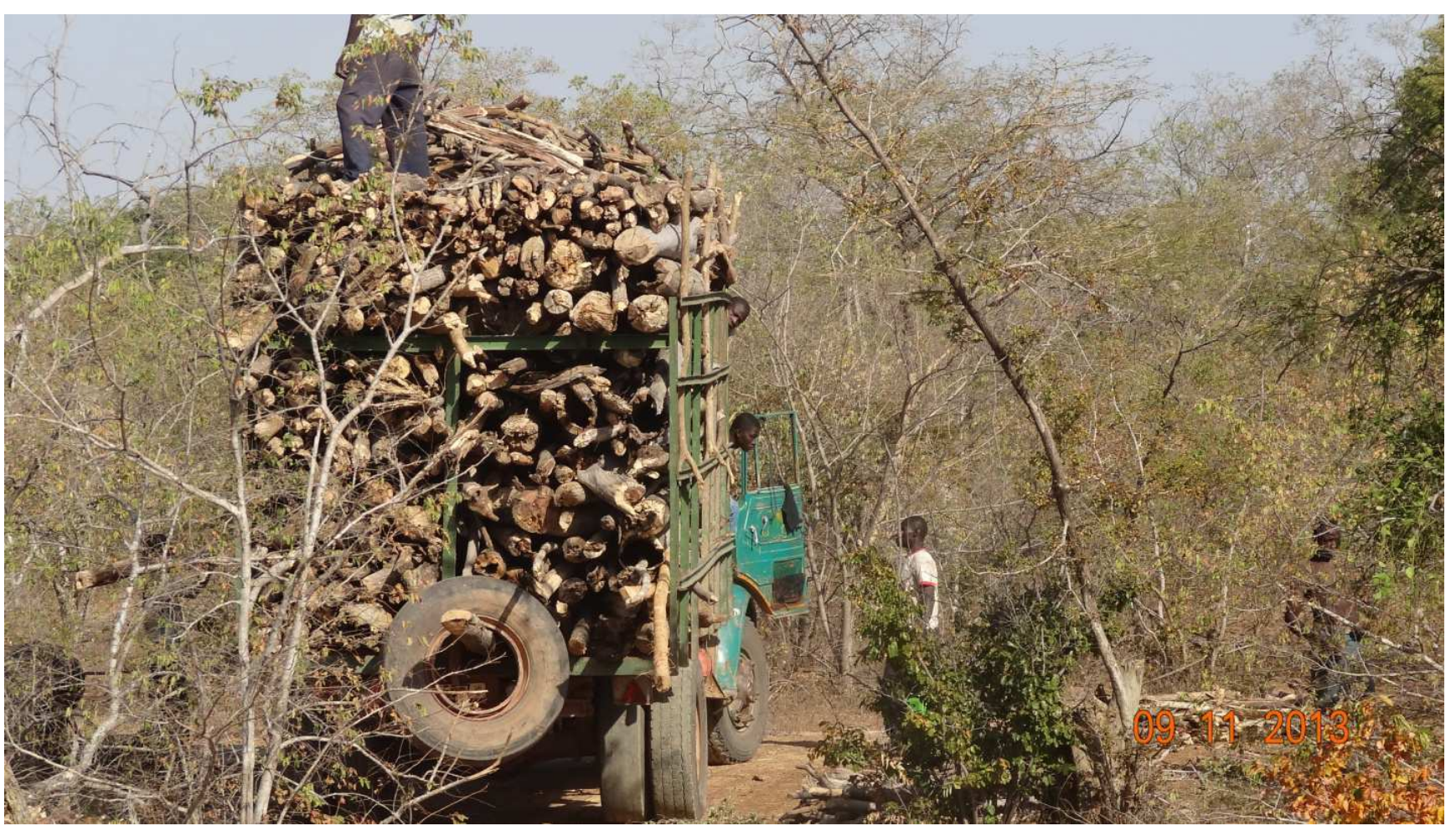

Photo 3 : Illustration du prélèvement du bois dans la forêt Classée de Tiogo (Tankoano, 2013). 


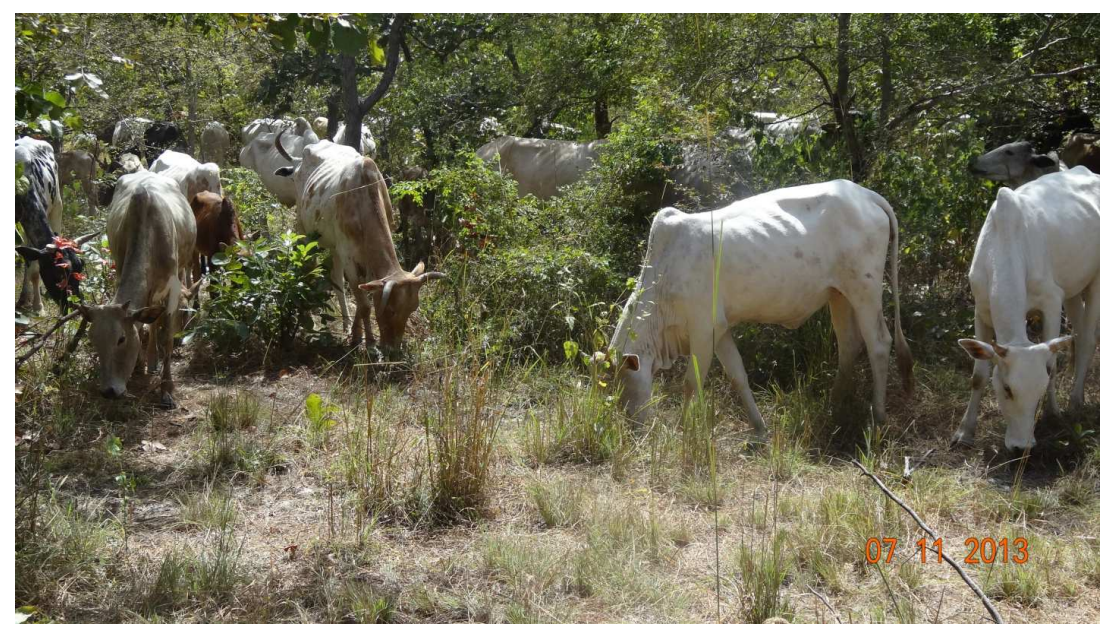

Photo 4 : Troupeau de bœufs en pâturages dans la forêt Classée de Tiogo (Tankoano, 2013).

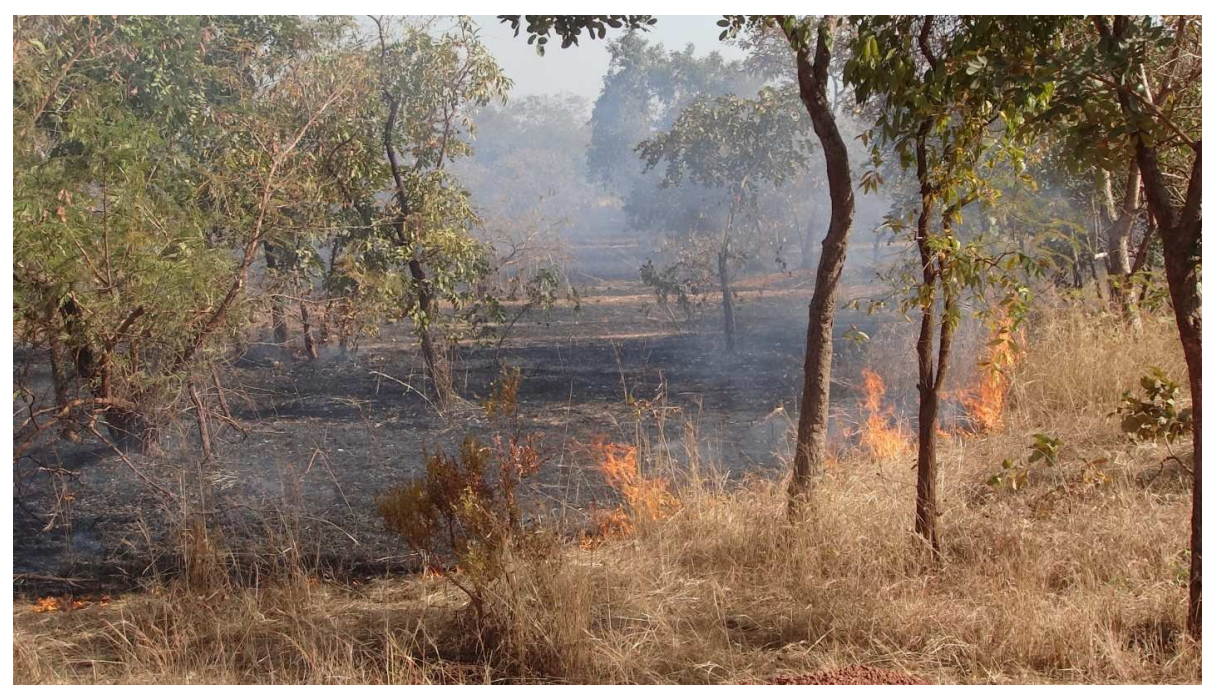

Photo 5 : Feux de brousse à l'intérieur de la Forêt Classée de Tiogo (Tankoano, 2013).

DISCUSSION

Traitement numérique et choix des indices de structure spatiale

Les classifications dirigées ont permis d'obtenir des précisions cartographiques satisfaisantes pour les deux images. Cela s'explique, d'une part, par la qualité des images et d'autre part, par le choix des classes de végétation et leur fusion (N'Guessan et al.,
2006 ; Inoussa et al., 2011). Les données de « vérité terrain » et la connaissance de la zone d'étude ont facilité également les analyses et l'interprétation visuelle des images. Malgré ces précisions cartographiques globalement satisfaisantes, les classes ne sont toujours pas très bien distinctes entre elles. Ces confusions sont dues à des réponses spectrales similaires pour certaines formations ligneuses (Koné et 
al., 2007; Diallo et al., 2011). En plus, les feux de brousse affectent la plupart des types de formations végétales qu'ils parcourent, rendant souvent complexe la discrimination entre les classes de végétation (N'Da et al., 2008; Diallo et al., 2011 ; Inoussa et al., 2011; Toko et al., 2012 ; Mama et al., 2013).

Dans notre étude, nous avons utilisé des indices de structure spatiale pour évaluer le degré de la fragmentation des savanes boisées. Ainsi, pour une meilleure analyse de la dynamique spatio-temporelle des taches de savanes boisées, les indices tels que le nombre de taches, l'aire moyenne, l'aire totale, l'indice de forme, la dominance, l'indice de Shannon et l'indice d'équitabilité de Piélou, ont été calculés. Le choix de ces indices est guidé par le fait que dans la littérature ils sont les plus fréquemment utilisés. Ainsi, plusieurs auteurs (Bamba et al., 2008; Kabulu et al., 2008 ; Barima, 2009; Inoussa et al., 2011 ; Diallo et al., 2011 ; Toko et al., 2012) ont utilisés avec succès les indices des structures spatiales pour quantifier les changements d'occupations du sol après classification des images satellites. L'existence de corrélation entre ces indices, même si elle indique une redondance de l'information (Bogaert et Mahamane, 2005), constitue un argument pour mettre en évidence le fait que les observations ne sont pas dues à un hasard.

\section{Dynamique des savanes boisées et activités anthropiques}

Les résultats obtenus montrent que dans la forêt classée de Tiogo, les savanes boisées sont en régression de 1986 à 2010. Cette régression pourrait être liée aux activités humaines (coupe de bois-énergie, agriculture, feux de brousse, surpâturage). L'anthropisation est marquée par l'augmentation des surfaces agricoles au détriment de la végétation entre 1986 et 2010. Ainsi, nous constatons une progression des surfaces agricoles de l'ordre de 4,38\% par an. La proximité de la forêt classée avec
Ouagadougou et la ville de Koudougou, en fait une réserve de bois-énergie. L'exploitation forestière serait la principale cause de la réduction des superficies de savanes boisées. Car, cette activité privilégie le prélèvement des arbres de gros diamètres. Or la majorité des individus de diamètres exploitables pour le bois énergie se concentrent dans cette formation végétale (savanes boisées). La coupe abusive et anarchique du bois a déjà été révélée comme un facteur important dans la dynamique régressive des ligneux, par plusieurs auteurs (Ozer, 2004; Faye et al., 2008; Tabopda, 2008). En plus, le passage régulier des feux ne favorise pas la reconstitution de la végétation naturelle (Diallo et al., 2011).

\section{Dynamique de la structure spatiale des savanes boisées et activités anthropiques}

L'analyse des cartes de répartition spatiale des taches de savanes boisées montre une dégradation accentuée de cette classe. La comparaison des deux cartes de répartition spatiale des savanes boisées montre un certain morcellement des taches de grande taille en des taches de petite taille. Les indices de structure spatiale confirment les transformations spatiales opérées dans cette formation forestière en 24 ans. Cela est illustré par une forte augmentation du nombre de taches de la classe ainsi que par une diminution aussi forte de l'aire moyenne des taches. Ainsi, l'analyse de la dynamique spatio-temporelle des savanes boisées a fait ressortir un processus de création de nouvelles taches pour cette classe et une réduction de leur superficie entre 1986 et 2010. Ce résultat est en accord avec celui de Touré (2010), qui dit que la régression de la superficie et l'augmentation des taches d'une classe signalent un processus de dégradation de ladite classe et par conséquent sa fragmentation. En outre, l'accroissement de la valeur de l'indice de Shannon met en relief une dislocation de l'unité (Touré, 2010). Cela 
signifierait que la classe a connu un grand morcellement des taches dominantes en des taches de petite taille. Cette forte fragmentation des savanes boisées pourrait s'expliquer principalement par leur exposition aux activités humaines telles que les défrichements agricoles, la quête permanente du bois-énergie et le surpâturage. Les activités anthropiques ont déjà été révélées comme un facteur important dans la dynamique régressive des formations végétales (Bamba et al., 2008; Faye et al., 2008; Kabulu et al., 2008 ; Inoussa et al., 2011 ; Toko et al., 2012). Mais, l'exploitation du bois-énergie et l'agriculture seraient les principales causes de cette fragmentation des savanes boisées. Car nous avons constaté une forte activité agricole et une exploitation quasi-quotidienne du bois au sein de la forêt classée. Arouna (2012) renchérit que les causes majeures de la fragmentation des forêts sont l'agriculture, l'exploitation forestière et la carbonisation par les populations locales. Quant à Bogaert et Mahamane (2005), l'anthropisation dans un milieu naturel est généralement caractérisée par une fragmentation, un changement dans la géométrie des taches et une évolution des classes anthropisées au détriment des classes naturelles d'occupation du sol. Ainsi, la fragmentation des savanes boisées aurait des répercussions graves sur la biodiversité de la forêt classée. En effet, la fragmentation entraîne la réduction d'un habitat continu de grande taille en habitats plus petits et isolés les uns des autres (Tabarelli et al., 2008). La diminution de la taille des fragments d'habitats et l'augmentation de leur isolement réduisent, à long terme, la viabilité des populations d'espèces végétales et animales qui y vivent de par la limitation, voire la disparition, des échanges entre ces populations, du fait de manque de connectivité (Cristofoli et Mahy, 2010). La fragmentation est une menace pour la conservation de la biodiversité, car une homogénéisation des paysages s'opère via l'agrandissement des espaces agricoles (Sabatier et al., 2010).

\section{Conclusion}

Cette étude avait pour principal objectif d'analyser la dynamique spatio-temporelle des taches des savanes boisées de la Forêt Classée de Tiogo à partir de l'imagerie satellitaire. Pour ce faire, nous avons utilisé les techniques de l'Ecologie du paysage couplées à la Télédétection et au Système d'Information Géographique. L'étude a révélé de fortes modifications du paysage de la forêt classée de Tiogo. La classe des savanes boisées, en l'occurrence, a connu une forte diminution. Ainsi, entre 1986 et 2010, ces savanes boisées ont perdu 5252 ha. Dans cette classe, de nouvelles taches ont été créées, entraînant une modification profonde de sa structure spatiale. Une des causes majeures de la fragmentation de cette classe serait liée à l'exploitation forestière et aux défrichements agricoles. Cependant, cette modification du paysage de la forêt classée de Tiogo n'est pas sans conséquences sur la conservation de la biodiversité. En somme, nous pouvons dire que l'imagerie spatiale couplée aux indices calculés a permis d'obtenir des indicateurs pertinents pouvant aider à la prise de décision en vue d'une gestion durable de cet écosystème forestier.

\section{CONFLITS D'INTERET}

Les auteurs déclarent qu'ils n'ont pas de conflits d'intérêt.

\section{CONTRIBUTIONS DES AUTEURS}

BT a conduit les travaux de terrain, l'analyse et les traitements des images satellitaires, des données de terrain et la rédaction du manuscrit. $\mathrm{MH}$ a initié cette étude et a pris part à la collecte des données de terrain, l'analyse des données, la discussion et la rédaction du manuscrit. N'HD a contribué aux traitements des images satellitaires, à la discussion des résultats et à la rédaction du 
manuscrit. ZS et JTY ont participé aux activités de terrain et à la lecture du manuscrit. IS a apporté un appui à la discussion des résultats et a apporté des corrections au manuscrit.

\section{REMERCIEMENTS}

Nos remerciements à l'Etat et surtout le contribuable Burkinabé pour nous avoir octroyé la bourse nécessaire à notre formation. Nos remerciements vont également au Service forestier chargé de la gestion de la Forêt Classée de Tiogo pour nous avoir facilités l'accès au site d'étude et aux populations riveraines de la Forêt Classée pour leur accueil chaleureux.

\section{REFERENCES}

Arouna O. 2012. Cartographie et modélisation prédictive des changements spatiotemporels de la végétation dans la commune de Djidja au Bénin : implications pour l'aménagement du territoire. Thèse de doctorat unique. Université d'Abomey-Calavi, Bénin, p.162.

Bamba I, Mama A, Neuba DFR, Koffi KJ, Traoré D, Visser M, Sissin B, Lejoly J, Bogaert J. 2008. Influence des actions anthropiques sur la dynamique spatiotemporelle de l'occupation du sol dans la province du Bas-Congo (R.D. Congo). Sciences et Nature, 5(1): 49-60.

Barima S. 2009. Dynamique, fragmentation et diversité végétale des paysages forestiers en milieux de transition forêtsavane dans le Département de Tanda (Côte d'Ivoire). Thèse de doctorat unique, Université Libre de Bruxelles, Belgique, p.178.

Bogaert J, Mahamane A. 2005. Ecologie du paysage: cibler la configuration et l'échelle spatiale. Annales des Sciences Agronomiques du Benin., 7(1) : 39-68.

Clerici N, Bodini A, Eva H, Grégoire JM, Dulieu D, Paolini C. 2007. Increased isolation of two Biosphere Reserves and Surrounding Protected Areas (WAP: WArly-Pendjari, Ecological Complex, West Africa). J. Nat. Conserv., 15: 2640

Cristofoli S, Mahy G. 2010. Restauration écologique: contexte, contraintes et indicateurs de suivi. Biotechnol. Agron Soc Environ., 14: 203-211.

Davidson C. 1998. Issues in measuring landscape fragmentation. Wildlife Soc. Bul., 26: 32-37.

Diallo H, Bamba I, Barima YSS, Visser M, Ballo A, Mama A, Vranken I, Maïga M, Bogaert J. 2011. Effets combinés du climat et des pressions anthropiques sur la dynamique évolutive de la végétation d'une zone protégée du Mali (Réserve de Fina, Boucle du Baoulé). Sécheresse, 22(3) : 97-107.

Direction des Forêts (DIFOR). 2007. Situation des forêts classées du Burkina Faso et plan de réhabilitation. Ministère de l'Environnement et du Cadre de Vie (MECV), Burkina Faso, p. 46.

Fahrig L. 2003. Effects of habitat fragmentation on biodiversity. Ann Rev. Ecol. Evol. Syst., 34: 487-515.

Faye E, Diatta M, Samba ANS, Lejoly J. 2008. Usages et dynamique de la flore ligneuse dans le terroir villageois de Latmingué (Sénégal). Journal des Sciences et Technologies, 7(1): 43-58.

Grégroire JM, Simonetti D. 2010. Interannual changes of fire activity in the Protected area of the SUN (Sustainable use of natural vegetation in West Africa) Network and other Parks and Reserves of the West and Central Africa Region derived from MODIS observations. Remote Sens, 2: 446-463.

Hien M, Boussim J, Guinko S. 2002. L'utilisation de Burkea africana Hook (Caesalpiniaceae) par les populations d'éléphants (Loxodonta africana Blumen Bach) dans le Ranch de Gibier 
de Nazinga (Burkina Faso). Ann. Bot. Afr. Ouest, 2: 25-35.

Inoussa MM, Mahamane A, Mbow C, Saadou M, Yvonne B. 2011. Dynamique spatiotemporelle des forêts claires dans le Parc national du $\mathrm{W}$ du Niger (Afrique de l'Ouest). Sécheresse, 22(2): 108-116.

Kabulu D J, Bamba I, Munyemba KF, Defourny P, Vancutsem C, Nyembwe NS, Ngongo LM, Bogaert J. 2008. Analyse de la structure spatiale des forêts au Katanga. Ann. Fac. Sc. Agro., 1(2): 12-18.

Koné M, Aman A, Yao ACY, Coulibaly L, N'Guessan KE. 2007. Suivi diachronique par télédétection spatiale de la couverture ligneuse en milieu de savane Soudanienne en Côte d'Ivoire. Télédétection, 7(1-2-3-4): 433-446.

Mama A, Sinsin B, De Cannière C, Bogaert J. 2013. Anthropisation et dynamique des paysages en zone soudanienne au nord du Bénin. Tropicultura, 31: 78-88.

Mbow C. 2009. Potentiel et dynamique des stocks de carbone des savanes soudaniennes et soudano- guinéennes du Sénégal. Thèse de Doctorat d'Etat, Université Cheikh Anta Diop de Dakar, Dakar, p. 319.

McGarigal K, Marks BJ. 1995. FRAGSTATS: Spatial Pattern Analysis Program for Quantifying Structure. Department of Agriculture, Pacific Northwest Research Station General Technical Report PNWGTR-351. Oregon, USA. (disponible sur le site internet http://www.fs. fed.us/pnw/pubs/gtr_351.pdf).

N'Da DH, N'Guessan EK, Wadja M. E, Affian K. 2008. Apport de la télédétection au suivi de la déforestation dans le parc national de la Marahoué (Côte d'Ivoire). Télédétection, 8(1): 1734.

N'Guessan E, Dibi N'da H, Bellan M, Blasco F. 2006. Pression anthropique sur une réserve forestière en Côte d'Ivoire. Télédétection., 5(4): 307-323.

Nouvellet Y, Sawadogo L. 1995. Recherches sur l'aménagement des formations naturelles dans la région du centre ouest du Burkina Faso. SUAS/CNRSTIRBET/CIRAD-Forêt, $88 \mathrm{p}$.

Ozer P. 2004. Bois de feu et déboisement au Sahel: Mise au point. Sécheresse, 15(3): 243-251.

Patton DR. 1975. A diversity index for quantifying habitat "edge". Wildlife Society Bulletin., 3: 171-173.

Sabatier R, Doyen L, Tichit M. 2010. Reconciling production and conservation in agrolandscapes : does landscape heterogeneity help? Innovation and Sustainable Development in Agriculture and Food (ISDA). Montpellier, France, $10 \mathrm{p}$.

Savadogo P. 2002. Pâturage de la forêt classée de Tiogo: diversité floristique, productivité, valeur nutritive et utilisation pastorale. Mémoire d'Ingénieur. IDR, UPB, Burkina Faso, p. 105.

Sawadogo L. 2009. Influence de facteurs anthropiques sur la dynamique de la végétation des forêts naturelles de la zone soudanienne du Burkina Faso. Thèse d'Etat. Université de Ouagadougou, p.142.

Tabarelli M, Lopes AV, Peres CA. 2008. Edge-effects drive tropical forest fragments towards and early successional system. Biotropica., 40: 657-661.

Tabopda WG. 2008. Les aires protégées de l'extrême -Nord Cameroun entre politiques de conservation et pratiques locales. Thèse de doctorat en Géographie-Aménagement-

Environnement, Université d'Orléans, p.322.

Tankoano B. 2013. Contribution de la télédétection et des SIG à 
l'aménagement et à la gestion durable des aires protégées du Burkina Faso: Cas de la forêt classée de Tiogo (Région du Centre-Ouest). Mémoire de Master en Télédétection et SIG/ Botanique et Foresterie, Université Félix Houphouët Boigny de Cocody-Abidjan (Côte d'Ivoire), p. 75.

Toko MI, Touré F, Toko II, Sinsin B. 2012. Indices de structures spatiales des îlots de forêts denses dans la région des Monts Kouffé. VertigO-la revue électronique en sciences de l'environnement [En ligne], Vol.12,
$\mathrm{N}^{\circ} 3$, mis en ligne le 16 novembre 2012, consulté le 04 janvier 2013. URL: http://vertigo.revues.org/13059, DOI: 10.4000/vertigo. 13059.

Touré F. 2010. Contribution de la Télédétection et des SIG dans l'étude de la dynamique des îlots de forêt dense dans la Forêt classée des Monts Kouffé et sa périphérie au Bénin entre (19862006). Mémoire de DESS en Production et gestion de l'information géographique. RECTAS. Campus Universitaire Obafemi Awolowo. IléIfè. Nigéria, p.69. 